\title{
Unmanned Aerial System for use in Environmental Monitoring of Water Body Wave Motion
}

\author{
Steven Lloyd \\ Underwater Acoustics Group \\ Loughborough University \\ Loughborough, UK \\ S.D.Lloyd@lboro.ac.uk
}

\author{
Paul Lepper \\ Underwater Acoustics Group \\ Loughborough University \\ Loughborough, UK \\ P.A.Lepper@lboro.ac.uk
}

\author{
Simon Pomeroy \\ Underwater Acoustics Group \\ Loughborough University \\ Loughborough, UK \\ S.C.Pomeroy@lboro.ac.uk
}

\begin{abstract}
This paper details the research conducted on an open source flight controller for the use of monitoring surface and wave motion of water bodies. Testing was preformed on an industrial FANUC robotic arm, where a Pixhawk 2 was tested using pre-preprogrammed circles of varying sizes to mimic the amplitude of sinusoidal waveforms. Results show good to excellent comparability between the circle radii programmed, and the calculated displacement from the Pixhawk's reported acceleration. This was achieved through the use of Fourier Transforms, filtering and integration of the acceleration logged by the Pixhawk during tests. Such a system is envisaged to be used in the reduction of flow noise a hydrophone experiences from surface deployments, where real time monitoring of the surface would raise and lower a deployed hydrophone in the water column to reduce or eliminate flow noise. Further to this, this system could be used for an early warning tsunami detection system, which could compliment systems already deployed, as well as being a cost effective solution for areas where no systems are currently in place.
\end{abstract}

Index Terms-Unmanned aerial vehicles, Underwater Technology, Acoustic sensors, Oceans, Remote monitoring, Remote sensing

\section{INTRODUCTION}

The advent of the Unmanned Aerial System (UAS) has provided researches and enthusiasts with a unique opportunity in environmental monitoring applications. Traditionally, sampling is performed in-situ, either manually or automatically, or via the use of remote sensing platforms, such as satellites and aircraft. Each method has their own inherent benefits and limitations; manually collecting samples, especially in contaminated areas, is risky for the person(s) involved, and there is a significant cost involved. Automated sampling systems will only collect samples at a singular point in space, and often requires expensive and/or difficult deployment strategies. Manned mobile aerial systems can be used to generate large data sets quickly, over large areas although only certain types of parameters can be obtained at a distance, and this method is costly to implement. Acquisition of, for example, imagery data is highly weather dependant, due to flight limitations and data quality resulting in a reduction in good data gathering opportunities. UAS systems however are generally more versatile, customisable and readily available at lower costs compared to traditional remote sensing methods i.e. manned aircraft or satellite technology. This has, in part, been down to the year on year growth of the UAS industry [1], resulting in lower cost UAVs and components. The inexpensiveness, availability and ability of UAS has seen large growth in a multitude of different areas: Water sampling [2], [3], blow hole sampling [4], air quality measurements [5], in air acoustic measurement [6], and underwater acoustic acquisition [7] are all applications for UAS in-situ sample collection, with the latter being the main interest of the authors. Areas not mentioned here surround the use of cameras for photogrammetry and similar fields, which survey from a distance and therefore are not further included in this paper.

The authors method of using a UAS to acoustically survey the underwater environment involves landing a UAS on the surface of a water body and deploying a hydrophone. This method elongates the time of deployment, compared to hovering and dipping the hydrophone, as the motors onboard the UAS are the main source of power consumption. While on the surface of the water body the UAS will be in a standby state; motors will be off, but the sensors on-board the flight controller are still generating data, which is taken advantage of in this paper. The data from the accelerometer can be used to determine wave heights while the GPS provides the location of deployment. The calculated displacement from the accelerometer is intended to be used in dynamically winching the hydrophone deployed in the water column by either retracting or reeling out cable to the hydrophone reducing the noise picked up from surface movement. The GPS, barometer, gyroscope and magnetometer could also be used to compliment the accelerometer, but that is beyond the scope of this paper.

This method of landing a UAS on the surface of a water body is a form of surface deployment and suffers from an effect known as for surface heave [8]. Figure 1 demonstates the issue with this form of deployment: The deployed UAS moves around on the surface due to wave motion, swell and ripples, moving the hydrophone beneath the surface with it through the water collumn. This introduces a noise signature onto the hydrophone, similar to flow noise. This acoustic signature is not a real and can induce significant parasitic noise signatures in the aquired data [8]. The noise caused by surface heave generally occurs in the subsonic range bellow $20 \mathrm{~Hz}$, and can be removed by the use of a high pass filter before the 


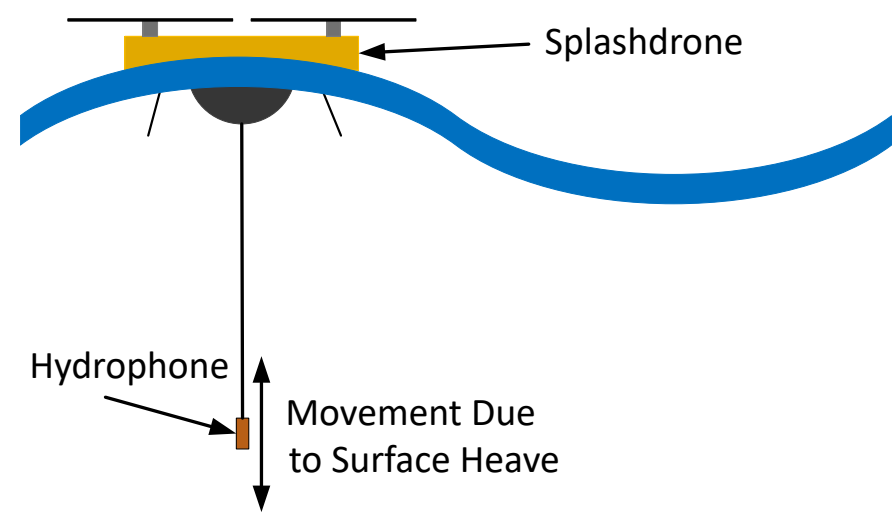

Fig. 1. Showing the effect surface heave has on a deployed hydrophone

Analogue to Digital Converter (ADC), to avoid saturation [8]. This method has a drawback: if frequencies of sub $10 \mathrm{~Hz}$ are of interest then this method is not feasible.

To combat this issue, conventional flow noise reduction techniques could be used on the UAS system to reduce the noise induced. One or a combination of the following methods could be employed:

Anti-heave syspension systems consist of a series of elasticated ropes/motion dampeners, which reduces the surface heave [8]. These motion dampeners increase payload weight, reducing flight time, and thus usability of the UAS. Sonar domes encase the area surrounding the deployed hydrophone, which is filled with water or oil to match the acoustic impedance of water. This drastically reduces flow noise, although adds significant weight to the system, which a UAS would not be able to handle. Sub-surface deployments, either located on the seabed, or close to it reduces the flow noise. Although research is on-going regarding submersible UAS, this technology is not suitable for purpose here.

The methods stated above do not apply to the current UAS technology level, they are either too heavy, or not practical when applied to a UAS based system. Instead, a new method (as stated above) is shown in this paper. The calibration of a Flight controller using a state of the art FANUC robotic arm is presented, which intends to show the feasibility of the proposed method under lab conditions.

\section{Comparable Systems}

Currently used water body sensor systems for measuring wave motion include wave buoys, weather station buoys, satellites, aircraft and wave radar. Wave/weather station buoys use sensors seen in UAS for measuring significant wave height, wave direction and wave energy spectra [9] [10]. Wave buoy systems are used for high-resolution data on small spatial scales, and are moored in order to keep them close to their original deployment position. Deployment of these devices requires the use of large ocean vessels to transport them to site, and tow boats for positioning. These devices are made for long term environmental monitoring, as they are slow and expensive to deploy and retrieve. The use of UAS technology for buoy monitoring systems has been achieved previously by Pedro Fernandes [11], where accelerometers and a GPS unit from a UAS where used to successfully measure wave heights. Although no experimental data has been found for this enthusiasts work, GPS was the main sensor used and, with the accelerometers as backup. Satellites are also an alternative, such as the Jason-3 satellite [12]. This satelite is part of the ocean surface topography mission, a collaboration between the USA and Europe. This satellite uses high frequency radar, along with GPS and a microwave radiometer to determine wave heights, sea level and ocean surface wind speeds [12]. It can do this for the entire globe every 10 days [12]. Satellites are expensive instruments but data is available for free from the Jason series of satellites (only one has real time data available [13]). Satellites are useful for long term, large area deployments, but due to the high speeds they travel at they can suffer from poor horizontal resolution $(300 \mathrm{~m}$ to $7 \mathrm{~km}$ depending on sample rate) [14]. Hydrones [15] present the idea of using UAS for water measurements, similar to the way satellites measure; using RADAR, though at distances much closer to the surface of the water body. The system has comparable results to a buoy nearby, although one main issue remains: readings are taken during flight. The system proposed in this paper would conserve the energy Hydrones system uses in flight by landing on water, thus allowing for longer deployments. The system proposed here also requires no additional sensors, meaning no loss in deployment time due to added system weight from the additional payload electronics. Autonomous Surface Vessels (ASV) are beginning to be used widely for a range of monitoring applications. They have the advantage that they can be deployed for extended periods of time, operating autonomously and often being selfpowered using wave, solar or wind power technology [16], [17], [18]. These systems have the ability to perform long term monitoring for a range of sensor packages. However, they lack the rapid deployment capabilities of combined flight and surface deployment as discussed in this paper. The concept of flight-based operation from unmanned surface ships is also under development by a number of researchers [19], [20], [21], which compliments the method presented in this paper.

\section{Methodology}

\section{A. System of Interest}

An open source flight controller platform, known as the Pixhawk 2 [22] was chosen for the research conducted in this paper. The Pixhawk can be programmed within ground control software to log specific sensor values, while ignoring integrated safety features which would have prevented arming the device and thus it being in a standby state. The system is comprised of two pieces, the Pixcube [22] which houses the main electronics, and the carrier board where external sensors, such as GPS modules and telemetry. The cube is $38.5 \mathrm{~mm}$ wide, $38.5 \mathrm{~mm}$ long and is $23 \mathrm{~mm}$ high. The carrier board is $94.5 \mathrm{~mm}$ long, $44.3 \mathrm{~mm}$ wide and 17.6 high at its highest point. Together the whole assembly measures $44.3 \mathrm{~mm}$ wide, $94.5 \mathrm{~mm}$ long and is $31.4 \mathrm{~mm}$ high. The cube has 29 Micro 
electromechanical systems (MEMS) based sensors on the onboard Inertial Measurement Unit (IMU) including three 3-axis accelerometers, three 3 -axis gyroscopes, three 3 -axis magnetometers and two barometers. There are three IMU in total, for redundancy purposes during flight [22]. Only one IMU was used throughout the experiments to keep results concurrent; all three would be used in the final system. These are logged to the on-board SD card at 30 times per second. It is possible to obtain real-time data out of one of the telemetry ports on the carrier board, which would be used in the final real-time system. Mission planner 1.3.49 build 1.1.6410.20232 was used for the set-up, accelerometer calibration and downloading of data flash logs from the Pixhawk 2. The Pixhawk 2 had APM: Copter firmware V3.5.3 Quad loaded onto it prior to all tests preformed.

\section{B. Manual Excitation}

The Pixhawk 2 was initially tested via manual excitation, and compared to the acceleration experienced during an indoor flight, in order to conclude if the acceleration produced during flight could be differentiated agaist. A Splashdrone Mariner 2 Auto version was equip with the Pixhawk 2 for testing of manual excitation and flight. For manual excitation, the Splashdrone and Pixhawk 2 system was attached to the end of a mobile platform anchored to an edge of a workbench. The UAS was then manually excited through $160 \mathrm{~cm}$ in the vertical direction, while the Pixhawk 2 logged sensor values. The system set-up along with axis orientation can be seen in Fig. 2. Post experiment, the data flash logs where downloaded to a PC through Mission planner, where the acceleration from the data flash logs where extracted using a custom LabVIEW script in LabVIEW 2015. The accelerometer sensor values where read into another custom script, this time in MATLAB 2016a, which filtered and integrated the acceleration to velocity, and again to displacement. The resultant displacement using all three axis where used as the motion experienced by the UAS was through three dimensions, rather than a single dimension per axis. This was repeated for the in-flight measurements of the UAS, which was performed indoors, to avoid influence from variable weather conditions.

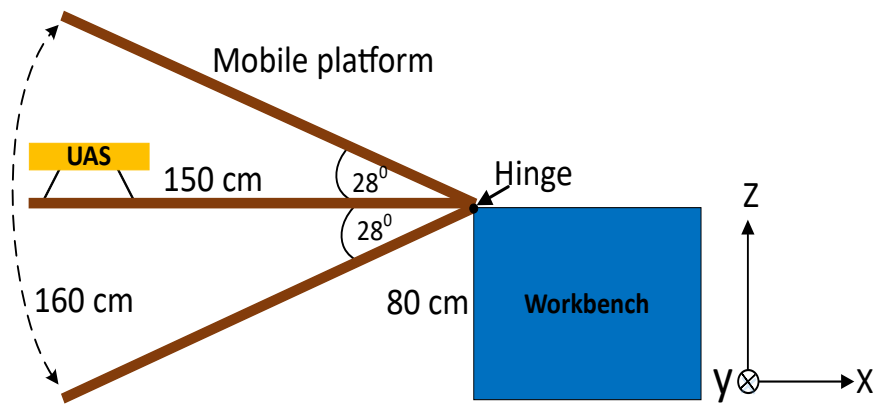

Fig. 2. Manual Excitation of the Pixhawk 2

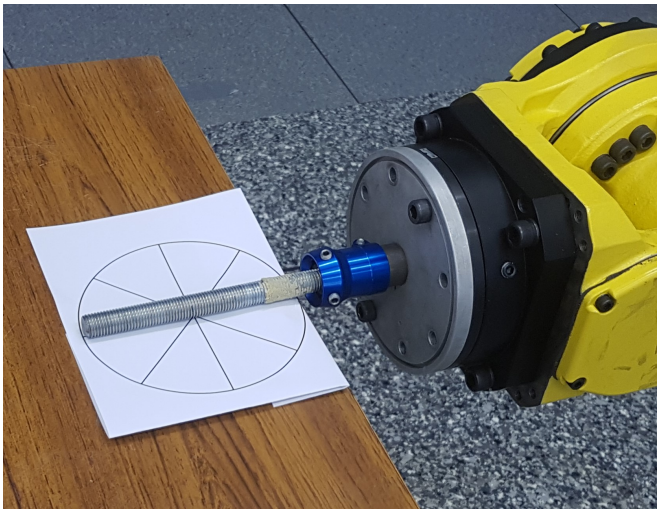

Fig. 3. Aiming method used for setting up the circular pattern on the FANUC

\section{FANUC Excitation}

After confirming the principle, the Pixhawk 2 was attached to a FANUC robotic arm for repeatability and multi-axis testing. The FANUC robotic arm model R-2000iC/165F was used which is capable of moving at $2000 \mathrm{~mm} / \mathrm{s}$, lifting 165 $\mathrm{kg}$, reaching $2655 \mathrm{~mm}$ and has 6 axes of rotation [23]. The FANUC arm was set to rotate in a circular manner, which excited two of the three axes of the accelerometer, at a multitude of different speeds and radii. The tests performed on the Pixhawk 2 are outlined in Table I.

The circular paths where set by using circular objects in space as reference points. The FANUC arm would be moved to 4 different positions close to $90^{\circ}$ away from one another, which is shown in Fig. 3

Each test preformed rotated in a clockwise direction, referenced to the facing direction of the Pixhawk 2 (Fig. 4). The programmed diameters for each circle used during tests can be seen in II, value of which come directly from the co-ordinates of the FANUC controller, which it calculated from the 4 points in space manually set. These values will be used as the circle sizes for calculations, although the circle sizes will be stated as 100,150 and $750 \mathrm{~mm}$ respectively for ease of reference.

Analysis of the acceleration logged during tests on the FANUC robotic arm where converted to displacement in MATLAB, via filtration and integration. The acceleration is initially placed through a Fast Fourier Transform (FFT) in MATLAB in order to identify the cut-off frequency $F_{C}$ for the Butterworth filters implemented. The mean is taken of the

TABLE I

TABLE CONTAINING THE TESTS PREFORMEd ON THE PIXHAWK 2

\begin{tabular}{|c|c|c|c|}
\hline Circle & $\begin{array}{c}\text { Speeds Tested } \\
(\mathrm{mm} / \mathrm{s})\end{array}$ & $\begin{array}{c}\text { Circle } \\
\text { Radii }\end{array}$ & $\begin{array}{c}\text { Axes } \\
\text { Excited }\end{array}$ \\
\cline { 1 - 3 } Circle 1 & $0,100,250$, & $0.375 \mathrm{~m}$ & $\mathrm{Z} \& \mathrm{Y}$ axis \\
\cline { 1 - 3 } Circle 2 & 500,750, & $0.075 \mathrm{~m}$ & $\mathrm{Z} \&$ Y axis \\
\cline { 1 - 1 } Circle 3 & 1000,1500 & $0.05 \mathrm{~m}$ & $\mathrm{X} \& \mathrm{Y}$ axis \\
\hline
\end{tabular}




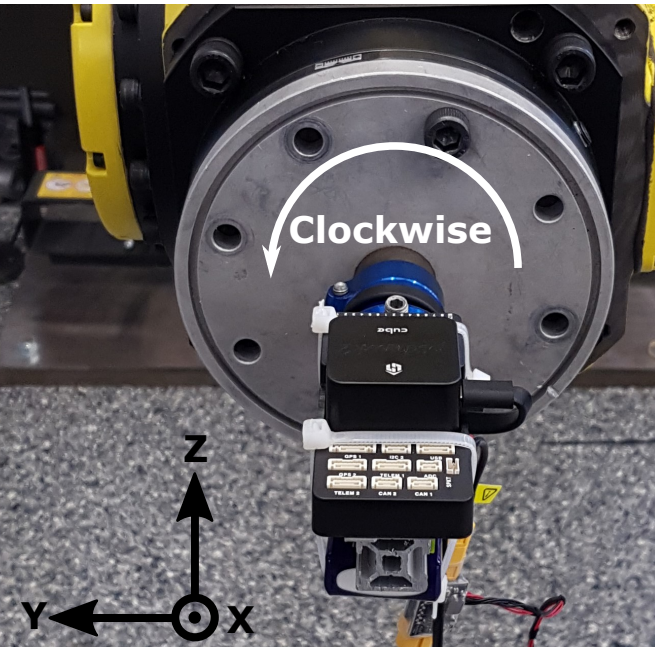

Fig. 4. Axis Directions of the Pixhawk 2 on the FANUC

TABLE II

Programmed Circle Sizes on the FANUC Robotic ARM

\begin{tabular}{|c|c|c|c|}
\hline \multicolumn{4}{|c|}{ Programmed Circle Diameters } \\
\hline Circle & X axis $(\mathrm{cm})$ & Y axis $(\mathrm{cm})$ & Z axis $(\mathrm{cm})$ \\
\hline Circle 1 & 0.76 & 75.32 & 74.86 \\
\hline Circle 2 & 1.04 & 10.25 & 10.01 \\
\hline Circle 3 & 14.62 & 14.83 & 0.10 \\
\hline
\end{tabular}

raw accelerometer data and is added back to the acceleration to remove any DC component present. The acceleration is then filtered via the use of a low pass and high pass filter, which are 4th order Butterworth filters having cut-off frequencies of $2 F_{C}$ and $0.5 F_{C}$ respectively. This is then integrated to obtain the velocity. The same method of DC removal, filtering and integration is used to obtain the velocity, and again for displacement.

\section{RESULTS}

\section{A. Manual Excitation}

Results from the manual excitation set-up shown in in Fig. 2 can be seen in Fig. 5. The $X$ and $Z$ axes experience the majority of acceleration, this is due to the arc that the UAS is manual excited through. Movement through an arc creates an acceleration perpendicular to the motion i.e. along the mobile platform, away from the hinge. The $\mathrm{Y}$ axis experiences some acceleration due to slight movements caused by the manual exciter. When compared to the acceleration experienced during flight (Fig. 6), both are noticeably different; it is possible to make out when the UAS is in standby, preparing to take off, flight and landing (see annotations on Fig. 6), whereas a clear waveform can be seen when manually exciting the Pixhawk 2 throug an arc. With these features, it is possible to distinguish between flight and surface deployment.
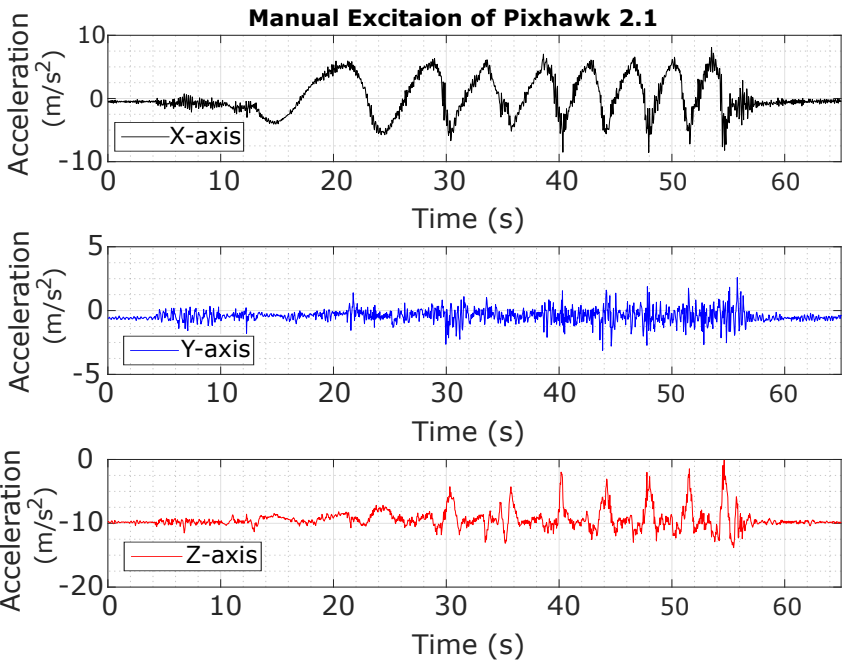

Fig. 5. Axis Directions of the Pixhawk 2 on the FANUC
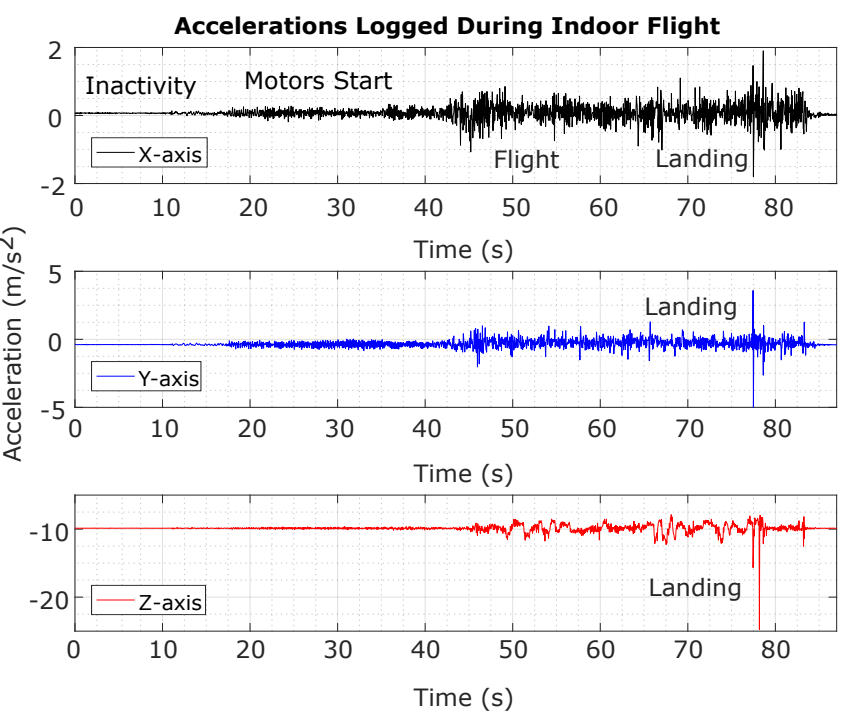

Fig. 6. Axis Directions of the Pixhawk 2 on the FANUC

From the manual excitation, the displacement over time can be seen in Fig. 7. The displacements are lower than expected, being in the order of $60 \mathrm{~cm}$ in each direction. This is likely down to the manual excitation of the experiment, rather than the accelerometers themselves.

These results show that it is possible to measure displacement, even through the manual excitation of the Pixhawk 2. In order to fully calibrate the system, the flight controller requires testing on the FANUC robotic arm, through a multitude of different tests. 


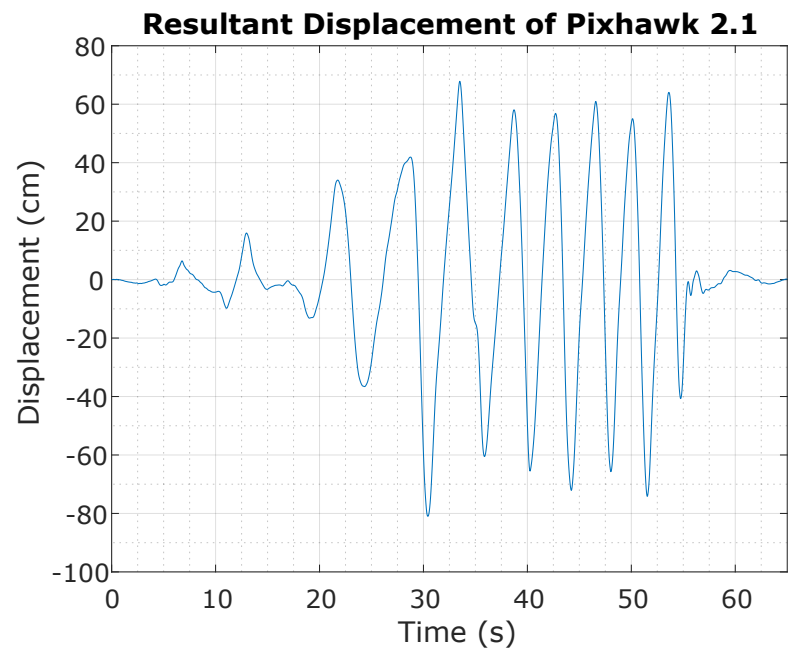

Fig. 7. Resultant Displacement from Manual Excitation of Pixhawk 2

\section{B. FANUC Excitation}

Figures 8, 9 and 10 show displacement snippets from the three FANUC circle tests preformed, at three different speeds. Each figure shows the two axis excited during testing, and cyan guidelines, which are the circle size guides plotted for ease of comparison. Snippets of data are shown as at the beginning of the tests the FANUC would need time to get up to speed, and stabilise its motion in the circular pattern programmed, this is similarly the case for end of the tests. Testing the Pixhawk on the small, $100 \mathrm{~mm}$ diameter circle pattern results in the highest angular acceleration, and therefore this is the most stressing of the tests for the on-board sensors. From Fig. 8 there appears to be a downwards trend of accuracy, the faster the FANUC is programmed to move. However; the results compare well at $100 \mathrm{~mm} / \mathrm{s}$ and $750 \mathrm{~mm} / \mathrm{s}$ respectively, both very near the $\pm 50 \mathrm{~mm}$ marks.

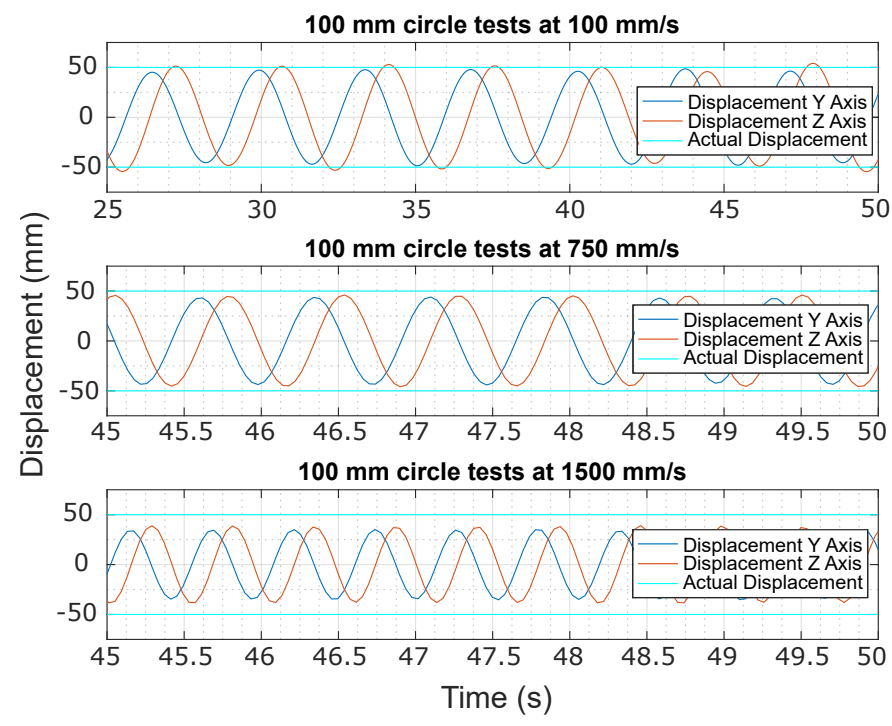

Fig. 8. Fanuc Testing $100 \mathrm{~mm}$ circle Tests
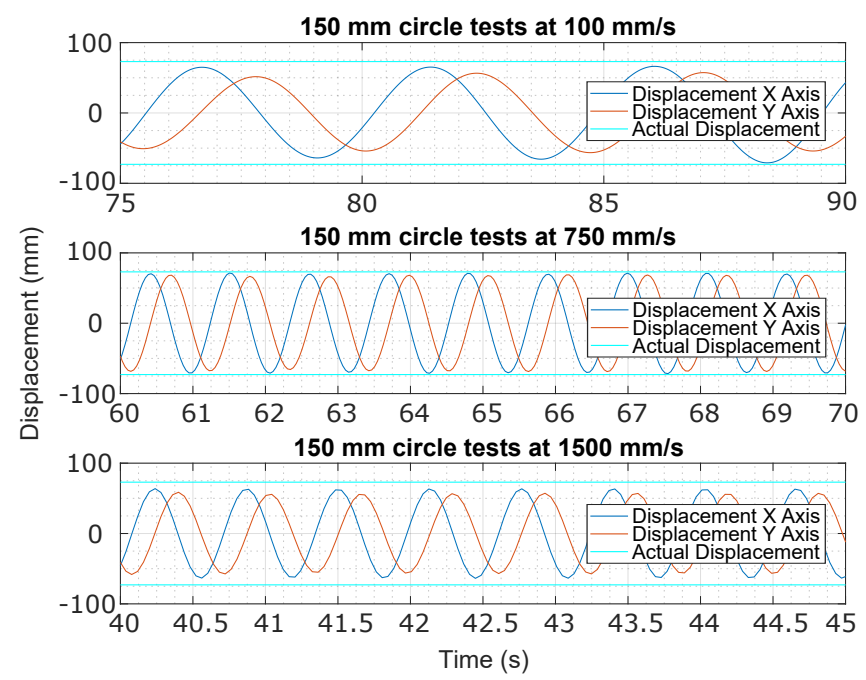

Fig. 9. Fanuc Testing $150 \mathrm{~mm}$ circle Tests

A slightly bigger circle diameter (Fig. 9 still shows a similar accuracy drop at higher speeds, although not as severe as the $100 \mathrm{~mm}$ circle tests, and this test tests different axis, which may explain the improvement. Again good comparability is maintained between the calculated displacement and the circle diameter for the slower tests.

The largest circle diameter used during tests (10 has good comparability between circle size and reported amplitudes for all three tests, which may come from the decreased acceleration experienced.

Testing has shown errors present which are shown numerically in table III. The displacement error tends to increase with speed, with larger dimensions being less affected.

Periods and frequencies from the Pixhawk tests can be seen in table $\mathrm{V}$, along with the calculated periods/frequencies, and the difference between them. Calculated periods and

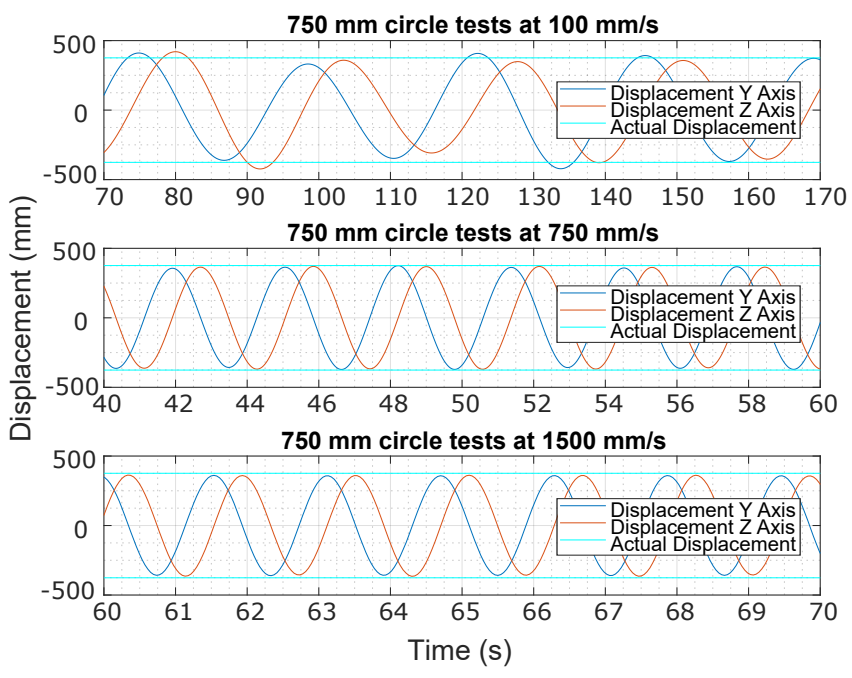

Fig. 10. Fanuc Testing $750 \mathrm{~mm}$ circle Tests 
TABLE III

DISPLACEMENT ERRORS COMPARISON

\begin{tabular}{|c|c|c|c|c|c|c|c|c|c|c|c|c|}
\hline & \multicolumn{6}{|c|}{ Displacement Errors (mm) } & \multicolumn{6}{|c|}{ Percentage Errors $(\%)$} \\
\hline Circle Size & \multicolumn{2}{|c|}{$100 \mathrm{~mm}$} & \multicolumn{2}{|c|}{$150 \mathrm{~mm}$} & \multicolumn{2}{|c|}{$1500 \mathrm{~mm}$} & \multicolumn{2}{|c|}{$100 \mathrm{~mm}$} & \multicolumn{2}{|c|}{$150 \mathrm{~mm}$} & \multicolumn{2}{|c|}{$1500 \mathrm{~mm}$} \\
\hline Speed & Y Axis & $\mathrm{Z}$ Axis & $\mathrm{X}$ Axis & Y Axis & Y Axis & Z Axis & Y Axis & $\mathrm{Z}$ Axis & $\mathrm{X}$ Axis & Y Axis & Y Axis & Z Axis \\
\hline $100 \mathrm{~mm} / \mathrm{s}$ & 7.0 & 0.1 & 14.2 & 36.2 & 1.8 & 30.4 & 6.9 & 0.1 & 9.8 & 24.5 & 0.2 & 4.1 \\
\hline $250 \mathrm{~mm} / \mathrm{s}$ & 6.6 & 2.9 & 4.1 & 11.6 & 16.5 & 21.5 & 6.5 & 2.9 & 2.8 & 7.8 & 2.2 & 2.9 \\
\hline $500 \mathrm{~mm} / \mathrm{s}$ & 10.6 & 6.1 & 5.0 & 12.9 & 20.1 & 15.0 & 10.4 & 6.1 & 3.4 & 8.7 & 2.7 & 2.0 \\
\hline $750 \mathrm{~mm} / \mathrm{s}$ & 15.1 & 9.0 & 7.3 & 17.0 & 30.2 & 17.4 & 14.8 & 9.0 & 5.0 & 11.5 & 4.0 & 2.3 \\
\hline $1000 \mathrm{~mm} / \mathrm{s}$ & 20.3 & 13.2 & 9.6 & 21.2 & 32.7 & 20.7 & 19.9 & 13.2 & 6.6 & 14.3 & 4.3 & 2.8 \\
\hline $1500 \mathrm{~mm} / \mathrm{s}$ & 32.7 & 23.9 & 19.8 & 34.7 & 41.0 & 27.5 & 32.1 & 23.9 & 13.6 & 23.5 & 5.4 & 3.7 \\
\hline
\end{tabular}

TABLE IV

SAMPLE RATE ACHIEVED DURING EACH TEST ON THE FANUC

\begin{tabular}{|c|c|c|c|}
\hline & \multicolumn{3}{|c|}{ Sample rate (Samples per Second) } \\
\hline Testing Speed $(\mathrm{mm} / \mathrm{s})$ & $100 \mathrm{~mm}$ Circle & $150 \mathrm{~mm}$ Circle & $750 \mathrm{~mm}$ Circle \\
\hline 100 & 24.94 & 24.94 & 24.94 \\
\hline 250 & 24.95 & 24.89 & 24.93 \\
\hline 500 & 24.94 & 24.90 & 24.88 \\
\hline 750 & 24.94 & 24.93 & 24.91 \\
\hline 1000 & 24.93 & 24.92 & 24.90 \\
\hline 1500 & 24.95 & 24.88 & 24.93 \\
\hline
\end{tabular}

frequencies where done via (1), where $\mathrm{f}$ is the Frequency $(\mathrm{Hz})$, $\mathrm{T}$ is the Period (s), D is the Diameter of the circles used during tests $(\mathrm{m})$ (taken from the programmed co-ordinates), and $\mathrm{S}$ is the speed at which the FANUC arm was programmed at $(\mathrm{m} / \mathrm{s})$.

$$
f=\frac{1}{T}=\frac{\pi D}{S}
$$

Finally, each tests' sample rate can be seen in Table IV, with all tests sampled at $30 \mathrm{~Hz}$ by the Pixhawk 2 .

\section{Discussion And Conclusions}

Testing the Pixhawk 2 via manual or robotic excitation has shown that the accelerometer, along with correct analysis, can monitor displacements. A comparison to Buoys used by the National Data Buoy Centre (NDBC) [25] can be made, where the buoys used by the NDBC are accurate to $+/-0.2 \mathrm{~m}$ over the range of 0 to $35 \mathrm{~m}$. The Pixhawk 2 error did not exceed 0.05 $\mathrm{m}$ during tests, however this was not out at sea in difficult condition, but the system shows promise non the less. At low speeds, differences in measured and actual displacements where low, at higher speeds the error observed becomes more significant, especially for smaller circle sizes. The increased error at higher speeds does not exclusively come from the accelerometer or analysis of the reported acceleration however; the FANUC arm and the settings used for testing are mainly at fault here. It can be observed that the FANUC is running slower than programmed, and is clearly visible by viewing the results in Table $\mathrm{V}$. The difference between the calculated period and frequency, when compared to the measured period and frequency by the Pixhawk, shows a trend of the error i.e. the FANUC is not operating at the pre-programmed velocity, it is operating at a slower rate. This may be down to the maximum rate of rotation being reached for the FANUC. In addition to this a setting used during the tests on the FANUC arm is believed to have caused the displacement error. The setting defined the behaviour of the arm when a point in space was set; either to go to and through the point set, or to try and go through said point, in order to maintain stability through the motions programmed. The second setting was used, which has resulted in the displacement errors seen in Fig. 8, Fig. 9 and Fig. 10.

Wind generates waves, and with this comes a limitation of the system; the UAS can fly in wind up to a point. The UAS used during tests (Splashdrone 2 Auto Version) can handle 17 mph wind speeds (Beaufort scale 4), whereas the Splashdrone 4 can handle up to $27 \mathrm{mph}$ (Beaufort scale 6). Choosing the Splashdrone 4 as reference, significant wave heights between 4-6 m [?], and wave periods in the order of 9 to 17 seconds [?] would be present. With this, the velocity that the UAS would be travelling at while on the surface of the water can be determined using (??), where $V_{S}$ is the velocity of the UAS, $H_{s}$ is the significant wave height and $T_{w}$ is the wave period. Taking the worst case (6 meter waves, 9.8 second periods), the UAS would be travelling at $1.3 \mathrm{~m} / \mathrm{s}$ vertically. The most probable sea state being Beaufort Scale 4, which means the UAS would be travelling at $0.8 \mathrm{~m} / \mathrm{s}$ (Wave height of 2.5 meters, period of 6 seconds). The Pixhawk 2 has been tested above these velocities, with smaller displacements; larger displacements suit the system better from results shown in this paper, therefore the Pixhawk 2 should be able to measure the wave heights used here.

$$
V_{S}=\frac{H_{s}}{T_{w}}
$$

Testing via sinusoidal excitation provided proof that the Pixhawk 2 can measure displacement, but a real world environment is complex; therefore future testing of this system in a real-world environment is required to establish how effective 
TABLE V

FANUC PERIODS AND FREQUENCIES MEASURED AND PROGRAMMED

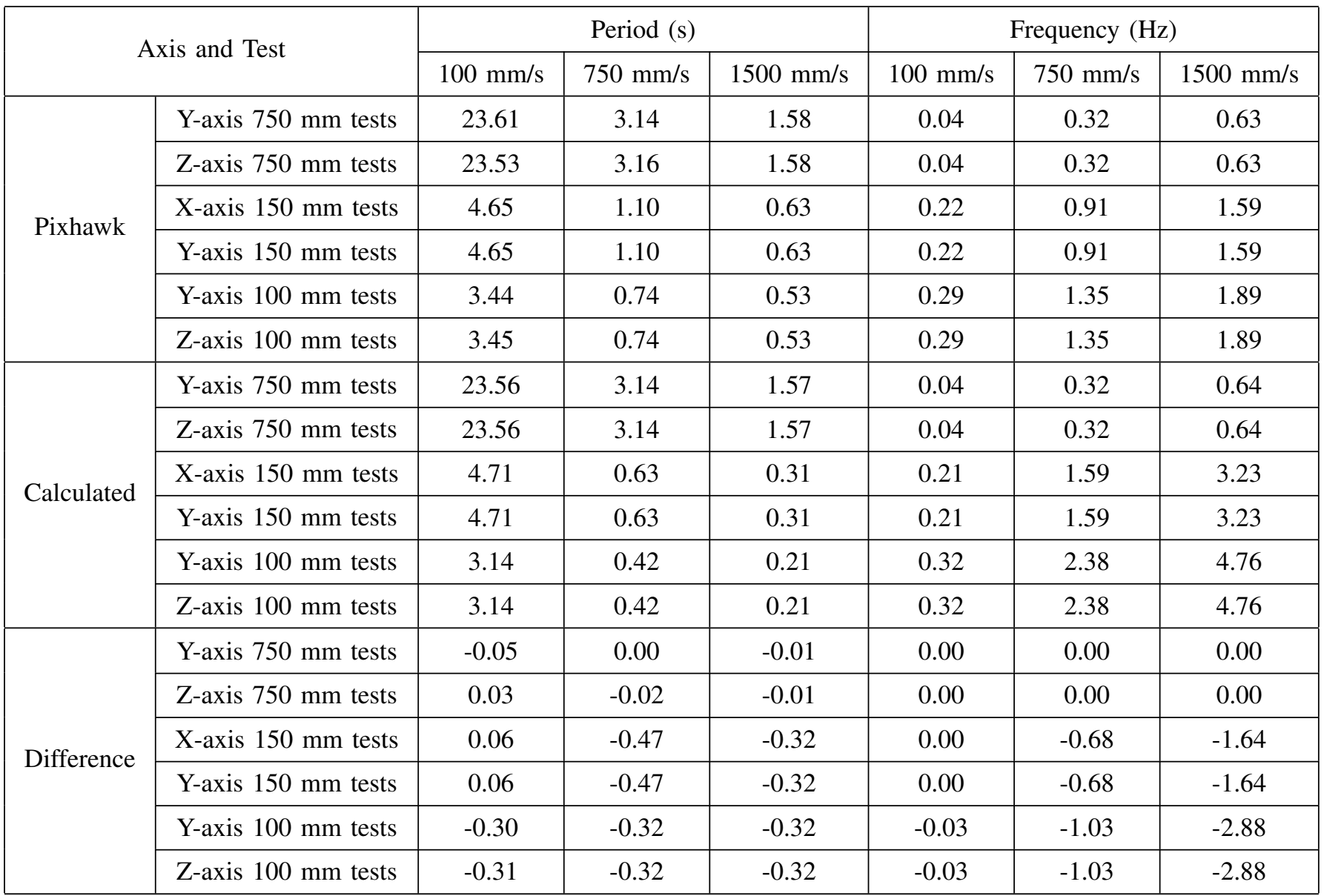

the system is at determining wave heights, which can be used to determine sea states. With regards to applications, an early warning detection system for tsunamis is a possible area. The system would detect the location of a Tsunami after an underwater earthquake has occurred, which would involve the use of disposable UAS on the land, which take off and land at a varying number of locations and distances out at sea. These UAS would be able to detect the incoming tsunami, and as the UAS has location data, the time between tsunami and it hitting land can be calculated to give people time to evacuate. This has the premise of being cheaper to maintain and deploy over the wave buoy counterparts. Another application, which is more relevant to the authors research, is a system which monitors wave heights to alleviate the symptoms of wave heave while acoustically surveying an area with a hydrophone. The waves move the UAS up and down which would cause the hydrophone to also move, which induces noise. This can be removed via filtering, although, as the occur at sub hertz range, any data that could be required is lost. By monitoring the wave height, the hydrophone can be moved via a motor up and down to counteract this heave, and reduce the noise induced onto the hydrophone.

\section{ACKNOWLEDGMENT}

We would like to thank James Jones for his guidance and training when using the FANUC robotic arm, and Stephen Carr for his advice and health and safety training.

\section{REFERENCES}

[1] D. Joshi, Commercial UAV Market Analysis 2017 2017-08-08, 2017. [Online]. Available: www.uk.businessinsider.com/commercialuav-market-analysis-2017-8?r=USIR=T. [Accessed: 07-Oct-2017].

[2] J.-P. Ore, S. Elbaum, A. Burgin, and C. Detweiler, Autonomous Aerial Water Sampling, J. F. Robot., vol. 32, no. 8, pp. 10951113, Dec. 2015.

[3] M. Ribeiro, A. S. Ferreira, P. Goncalves, J. Galante, and J. B. de Sousa, Quadcopter platforms for water sampling and sensor deployment, in OCEANS 2016 MTS/IEEE Monterey, 2016, pp. 15.

[4] A. Bennett, D. Barrett, V. Preston, J. Woo, S. Chandra, D. Diggins, R. Chapman, A. Wee, Z. Wang, M. Rush, and I. Kerr, Autonomous vehicles for remote sample collection: Enabling marine research, in OCEANS 2015 - Genova, 2015, pp. 18.

[5] T. Villa, F. Gonzalez, B. Miljievic, Z. Ristovski, and L. Morawska, An Overview of Small Unmanned Aerial Vehicles for Air Quality Measurements: Present Applications and Future Prospectives, Sensors, vol. 16, no. 7, p. 1072, Jul. 2016.

[6] S. A. Cerwin, Airborne collection of acoustic data using an unmanned aerial vehicle, 2005.

[7] S. Lloyd, P. Lepper, and S. Pomeroy, Evaluation of UAVs as an underwater acoustics sensor deployment platform, Int. J. Remote Sens., pp. 110, Nov. 2016. 
[8] S. P. P. Robinson, P. a. Lepper, and R. a. Hazelwood, Good Practice Guide for Underwater Noise Measurement, National Measurement Office, Marine Scotland, The Crown Estate. NPL Good Practice Guide No. 133, 2014.

[9] National Oceanic and Atmospheric Administration, NDBC Moored Buoy Program. [Online]. Available: http://www.ndbc.noaa.gov/mooredbuoy.shtml. [Accessed: 19-May2017].

[10] Glossary: Environment, land and water. [Online]. Available: https://www.qld.gov.au/environment/coasts-waterways/beach/wavesglossary/wave-buoy. [Accessed: 18-May-2017].

[11] P. Fernandes, UAV technology used for buoy monitoring system - quot;water dronesquot; - DIY Drones. [Online]. Available: http://diydrones.com/profiles/blogs/uav-technology-used-for-buoymonitoring-system-water-drones. [Accessed: 27-Sep-2017].

[12] Jason-3 Satellite - Mission. [Online]. Available: https://www.nesdis.noaa.gov/jason-3/mission.html. [Accessed: 27Sep-2017].

[13] GDR (Geophysical Data Records). [Online]. Available: https://www.aviso.altimetry.fr/index.php?id=1458. [Accessed: 27Sep-2017].

[14] Sea Height. [Online]. Available: http://www.ospo.noaa.gov/Products/ocean/ssheight.html. [Accessed: 27-Sep-2017].

[15] G. Valladeau, J. C. Poisson, O. Lauret, and P. Prandi, HYDRONES by CLS.

[16] AUTONAUT FOR PAM PASSIVE ACOUSTIC MONITORING.

[17] ] C-Worker $7 . \quad$ [Online]. $\quad$ Available: https://www.asvglobal.com/product/c-worker-7/. [Accessed: 07-Oct2017].

[18] C-Enduro. [Online]. Available: https://www.asvglobal.com/product/cenduro/. [Accessed: 07-Oct-2017].

[19] E. E. Moreland, M. F. Cameron, R. P. Angliss, and P. L. Boveng, Evaluation of a ship-based unoccupied aircraft system (UAS) for surveys of spotted and ribbon seals in the Bering Sea pack ice, J. Unmanned Veh. Syst., vol. 3, no. 3, pp. 114122, Sep. 2015.

[20] J. W. Durban, H. Fearnbach, W. L. Perryman, and D. J. Leroi, Photogrammetry of killer whales using a small hexacopter launched at sea, J. Unmanned Veh. Syst., vol. 3, no. June, pp. 15, 2015.

[21] L. C. Veloso and V. J. A. S. Lobo, Tests of a light UAV for naval surveillance, pp. 14.

[22] Pixhawk 2 (TheCube). [Online]. Available: http://www.proficnc.com/. [Accessed: 01-April-2019].

[23] FANUC R-2000 iC 165F. [Online]. Available: https://www.fanuc.eu/uk/en/robots/robot-filter-page/r-2000-series/r2000ic-165f. [Accessed: 01-May-2019]

[24] S. L. BALES, DESIGNING SHIPS TO THE NATURAL ENVIRONMENT, Nav. Eng. J., vol. 95, no. 2, pp. 3140, Mar. 1983.

[25] National Data Buoy Center (NDBC), 2005. [Online]. Available: http://www.ndbc.noaa.gov/rsa.shtml. [Accessed: 07-Mar-2018]. 